\title{
Match Running Performance During Fixture Congestion in Elite Soccer: Research Issues and Future Directions
}

\author{
Christopher Carling - Warren Gregson • \\ Alan McCall $\cdot$ Alexandre Moreira • \\ Del P. Wong • Paul S. Bradley
}

Published online: 19 February 2015

(c) Springer International Publishing Switzerland 2015

\begin{abstract}
It has been proposed that match congestion in elite soccer results in residual fatigue and underperformance in ensuing competition due to insufficient recovery time. In this article, matters relating to match congestion and running performance in elite soccer competition are discussed. We suggest a need to determine the extent to which elite players are, in reality, exposed to periods of match congestion and hence to potential declines in performance. Despite evidence of exercise-induced muscle damage combined with a decline in physical performance up to $72 \mathrm{~h}$ post-match, research using time-motion analyses suggests that running performance represented by distances
\end{abstract}

C. Carling

Institute of Coaching and Performance, University of Central

Lancashire, Preston, Lancashire, UK

C. Carling $(\bowtie) \cdot$ A. McCall

LOSC Lille Métropole Football Club, Domain de Luchin,

Camphin-en Pévèle, 59780 Lille, France

e-mail: christopher.carling@gmail.com

C. Carling · D. P. Wong

Human Performance Laboratory, Technological and Higher

Education Institute of Hong Kong (THEi), Hong Kong, China

W. Gregson

Football Exchange, Research Institute for Sport and Exercise

Sciences, Liverpool John Moores University, Liverpool, UK

\section{A. McCall}

Research Department for Sports and Exercise Science,

Napier University, Edinburgh, UK

\section{A. Moreira}

School of Physical Education and Sport, University of Sao

Paulo, Sao Paulo, Brazil

P. S. Bradley

Carnegie School of Sport, Leeds Beckett University, Leeds, UK covered is unaffected over periods of match congestion. We recommend analysis of alternative movement variables including accelerations, decelerations and turns that are taxing metabolically and contribute greatly to muscle damage. Moreover, a holistic approach combining subjective ratings with biochemical, hormonal and immunological responses to exercise would be pertinent, especially in players frequently exposed to match congestion. Contemporary practitioners typically implement various postmatch recovery treatments during dense schedules in an attempt to accelerate recovery and ensure that subsequent running performance is not unduly affected. However, empirical evidence to support their efficacy in maintaining running performance is lacking and we recommend controlled intervention studies using match simulations in an attempt to verify their effectiveness. These points are critically addressed using findings from the current scientific literature, while gaps in the current body of knowledge and future directions for research are highlighted.

\section{Key Points}

This paper suggests a need for research to determine the extent to which elite senior soccer players are actually exposed to periods of fixture congestion.

Modifications in the design of time-motion studies are recommended to establish whether players' match running profiles are in reality compromised during intensified competition periods.

Controlled intervention studies using match simulations are also recommended to verify the efficacy of post-match recovery modalities on competitive running performance. 


\section{Introduction}

In contemporary elite soccer, players should be able to compete in up to 50 matches per season and participate in a match every 4 days over a five-match period [1]. Periods of fixture congestion occur in elite soccer, notably for toplevel teams that compete for domestic league championships and cups in addition to European competitions. An accumulation of matches over a short period potentially results in residual fatigue and underperformance due to insufficient time for physical recovery whilst also increasing the propensity of injury [2]. Effective player management and monitoring by coaching and medical staff over intensified competitive schedules is unquestionably important. However, the extent to which players are in reality exposed to periods of fixture congestion and to a subsequent risk of a decline in ensuing match performance is unclear.

Research across various playing standards demonstrates the occurrence of muscle soreness during the post-match period, which is indicative of exercise-induced microtrauma and a concomitant elevation in muscle damage [3]. These morphological changes within muscles are accompanied by marked declines in field-test measures of anaerobic performance as well as repeated high-speed running performance for up to $72 \mathrm{~h}$ post-match [4-8]. In theory, these declines would negatively impact on ensuing competitive physical performance if the time delay between successive matches was short. Yet several time-motion analysis studies conducted on elite senior soccer competition have shown that external workload represented by distance covered at various running speeds was unaffected over a congested period [2, 9-15]. Despite the physiological trauma and accumulative fatigue potentially induced by successive matches, it would seem players recover sufficiently and cope 'physically' over a congested period. However, recent research suggests a need to modify current time-motion study designs and metrics to determine whether declines in running performance actually occur during congested competition periods [16, 17].

Practitioners working incontemporary elite soccer typically implement post-match recovery modalities (e.g., cold-water immersion, massage, compression garments) during dense fixture schedules in an attempt to accelerate recovery and ensure that performance in subsequent competition is not unduly compromised [18]. Several authors have suggested that such treatments aided in maintaining match running performance during congested periods $[2,9$, $10,14,15]$. However, limited empirical evidence currently exists to support the efficacy of post-match recovery modalities for attenuating potential decrements in running performance across consecutive matches played within a tight timeframe.
In this article, we discuss the extent of the problem of exposure to fixture congestion on competitive running performance. Suggestions are also made regarding both modifications to research design in time-motion studies and the need for experimental work to support the efficacy of post-match recovery treatments on subsequent match running performance during periods of fixture congestion. These points are critically addressed using information from the current scientific literature. Likewise, gaps in the current body of knowledge and future directions for research are highlighted.

\section{Is Fixture Congestion Actually a Concern?}

Coverage of elite player rotation strategies during the season and specifically during periods of fixture congestion has been neglected in the literature. Information is necessary to identify how frequently individual players actually participate in and complete matches in their entirety over short and/or prolonged congested periods. An investigation of an elite soccer team during a prolonged period of match congestion (eight matches in 26 days) reported a single outfield player who completed every match while six players took part in every match as either starters or substitutes [10]. In three separate congested periods (six matches in 18 days) across the same season, only four players in an elite team participated in every match [15]. Finally, analysis of 22 elite English teams who played three matches in 5 days yielded complete datasets for 16 outfield players [11].

The apparent limited extent to which individual players repeatedly completed entire matches across congested periods raises doubts about the real-world magnitude of the issue of match congestion and questions the value of the existing time-motion research in this area. The majority of clubs purely participate in domestic competitions but typically still use a selection of reserve players for cup competitions. Teams participating in European competition are also known to field reserve players for both domestic and European cups. When combined with the possibility of early elimination from cup competitions, these factors can ensure that some teams and players are rarely confronted by match congestion, especially over prolonged periods. A potentially greater concern for clubs in this case is ensuring that regularly non-selected players maintain match fitness and motivation!

It would seem from the limited number of players completing matches in the research mentioned earlier that coaching practitioners recognise the need to rotate players. However, it must be recognised that rotation can also be forced due to time-loss injury, illness and player suspensions [10]. Unfortunately, no factual information is 
currently available describing the reasons behind player selection policies during congested fixture periods. Complementary information from interviews conducted with coaching practitioners regarding player selection criteria would be pertinent (e.g., choice based on subjective opinions, physiological/physical monitoring training/matchplay data, prior number of matches played for their club and/or national team, tactics).

\section{Concerns with Time-Motion Analysis Research}

\subsection{Study Design}

Inferential statistical analyses of data derived from timemotion analyses generally demonstrate that match-tomatch measures of running performance in elite senior players do not vary statistically, even when successive matches are separated by minimal recovery time (Table 1 ). For example, no statistically significant changes in running performance were reported during both short (e.g., three matches in 5-7 days) $[9,11]$ and longer periods of congestion (e.g., eight matches in 26 days) [10, 15]. According to these apparently stable match running activity profiles, one could assume that elite players are simply able to maintain physical performance across congested schedules. Partly supporting this latter point are findings from a study of an Italian team competing at the highest level domestically [19]. Tests conducted following match-play showed that players fully recovered their physical capacities $48 \mathrm{~h}$ following competition. While larger-scale research is required, these findings suggest that elite players recover quicker than other ages and standards (up to $72 \mathrm{~h}$ ) and in theory are able to perform at 'normal' levels if the delay between games is $48 \mathrm{~h}$ or greater.

Alternatively, it could be that the time-motion measures previously employed are potentially lacking sensitivity or are simply invalid for determining whether 'fatigue' actually occurs. The first study investigating the influence of successive matches over a short time period on running performance in elite senior players was conducted on data collected from the 2005-2006 season [11]. Match running performance metrics used for inter-match comparisons in this first study and typically in later papers include total distance and distance covered across a range of running speeds at arbitrarily predefined speed thresholds, frequency of and mean recovery time between high-speed efforts, and peak running speed [2,9-13]. However, doubts have recently been cast [20] on the practical utility of these metrics and, notably, assessments of changes in simplistic measures such as distances covered that are commonly used in an attempt to identify the occurrence of declines in physical performance. Yet, at the time of writing, the latest published research in this area has also primarily employed analyses of distances covered [14, 15]. Furthermore, only two studies, again mainly using distances covered, have investigated whether match-related fatigue evolves across intensified competition periods by examining declines across halves [10, 12], while only one has examined changes related to playing position [14].

Another key issue when attempting to interpret the effects of fixture congestion is the 'natural' variation in running activities, notably at high speeds across successive matches and the competitive season [21]. The large variability in high-speed running is mediated through the inherent demands of match-play that are influenced by a myriad of performance-related and contextual factors that have not yet been accounted for in the aforementioned literature on fixture congestion. Factors include team formation and tactics, time spent in ball possession, effective playing time, individual fitness characteristics, and contextual issues such as result and playing environment [20]. Self-regulation or 'pacing' of physical efforts has also been suggested as a means employed by elite players to modulate and adequately respond to match demands during periods of fixture congestion [10-12], although using timemotion data alone to determine if a pacing strategy is adopted is unfeasible [22]. Collectively, these factors make it difficult to interpret inter-match changes in player running activity that may be associated with fatigue.

The inclusion of percentage coefficient of variation (\% $\mathrm{CV})$ values and confidence intervals would have provided an idea of variability for time-motion data previously collected during periods of fixture congestion. For example, a repeated-measures analysis of variance test revealed that high-speed running activity in an elite team as a whole for a series of eight matches played over a 26-day period revealed no significant differences across matches [10]. For the same data, a \% CV value of $\sim 30 \%$ was reported, whereas a substantially lower $\% \mathrm{CV}$ of $\sim 10 \%$ was obtained for data collected in matches played across the entire season in the same team. This larger match-to-match variability could have masked any 'real' changes in running performance across the congested period. The magnitude of the \% $\mathrm{CV}$ can inform whether a real change in performance has occurred rather than this being attributable to 'normal' between-match variation [21]. Indeed, there is a need generally for consensus between practitioners and researchers on what changes in running performance can be considered 'meaningful' [20] and, for the present purposes, potential declines in running activity related to match congestion.

In future studies, systematic inclusion of effect-size values would provide practical interpretation of the magnitude of the differences across consecutive matches. For example, no statistical difference in mean high-speed 


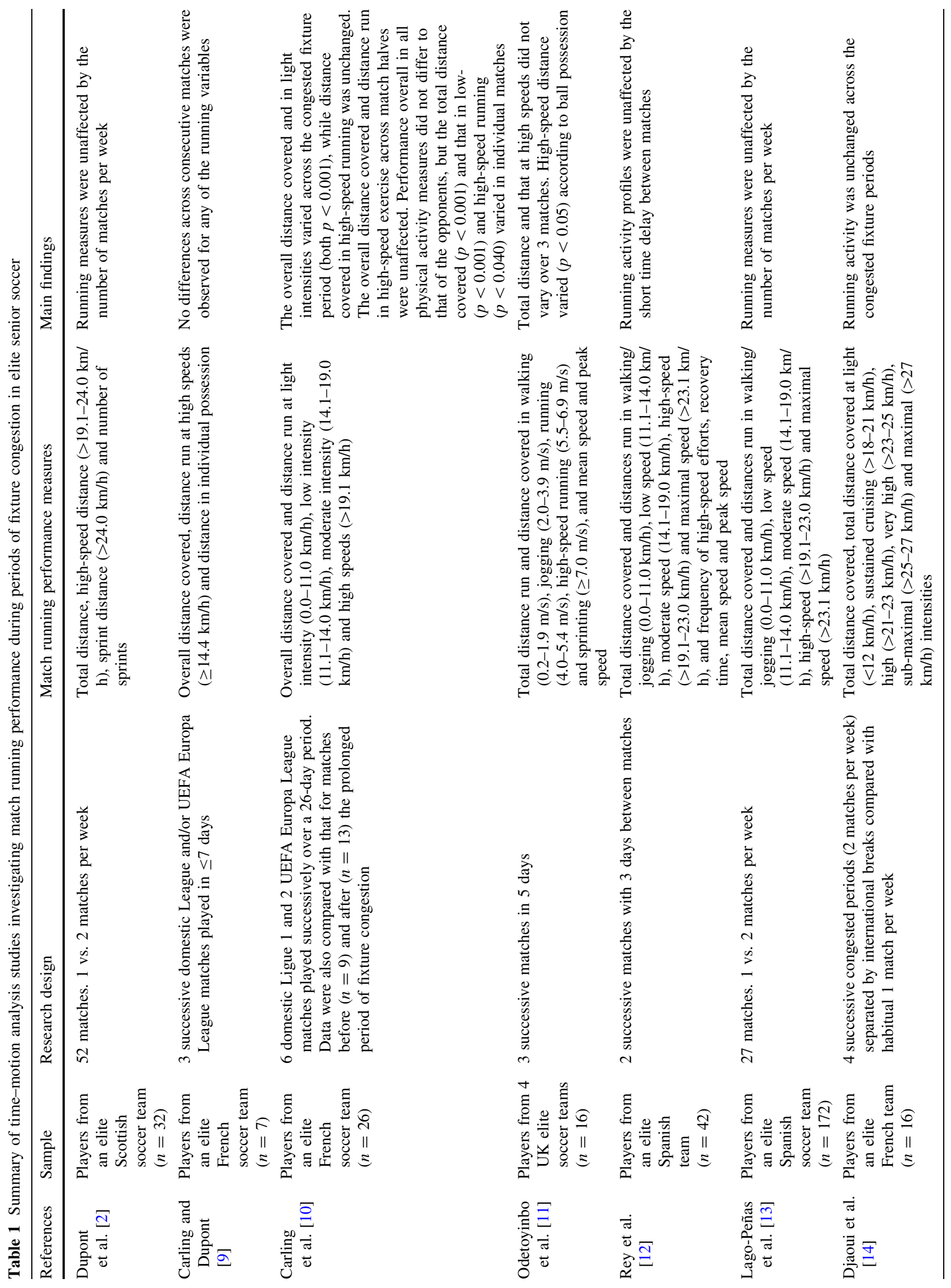


running distance was reported in the same players across three matches played in $\leq 7$ days $(2,667 \pm 200$ vs. $2,629 \pm 398$ vs. $2,414 \pm 145 \mathrm{~m}$ ) [9]. Yet, the effect-size values for matches 1 and 2 versus match 3 were -0.72 and -1.45 suggesting moderate and large declines, respectively [23].

In addition to the complexities of data interpretation due to match-to-match variability, there are additional methodological issues that require consideration. For example, several published papers have collected data from a single club setting, thereby only reflecting performance of the team in question. Although small-scale case study research that addresses specific performance-related questions is important [24], larger-scale investigations (more match observations, players and teams) are necessary to enable detection of more meaningful changes and generalised trends given the highly variable nature of match performance metrics [21]. The analysis of a single team is also problematic in that several studies on match congestion have been unable to verify changes in performance across playing positions and/or in individual players. As mentioned earlier, these low sample sizes can be linked to player rotation, time-loss injuries, illnesses and suspensions. To partly counter this problem, one study included time-motion data from players who had participated in a minimum of $75 \mathrm{~min}$ of play [15]. However, the lack of information on the final $15 \mathrm{~min}$ of matches, in which there can be substantial reductions in running activity [20], might have influenced the findings and contributed to the reported lack of match-to-match changes in running performance.

\section{Future Directions for Time-Motion Analysis Research}

Methodological improvements, notably in terms of the variables analysed and the general approach to research designs, are recommended if future investigations into the effects of match congestion on player performance are to be more meaningful. These points and prospective avenues of research are discussed in turn.

\subsection{Additional Running Performance-Related Analyses}

As mentioned earlier, time-motion analyses tend to monitor changes in measures of distances covered, notably at high speeds using arbitrarily predefined speed thresholds. However, players differ greatly in the speed at which they begin to run at high speeds. Accounting for physiological capacity to individualise the high-speed threshold could provide a more valid and sensitive method for quantifying match-to-match changes in activity [25], especially as fixture congestion has been linked to impaired 
intermittent field-test running performance [8]. A combination of players' anaerobic threshold, maximal aerobic speed and maximal sprinting speed characteristics are considered necessary to individualise and improve interpretation of time-motion data [26].

In reality, however, there are practical challenges to administering this individualised approach and unfortunately it does not account for the high metabolic cost of typical utility movements [25]. Metabolic power estimations adjust data to incorporate the additional energy cost of acceleration and deceleration activities [27]. Although no studies have yet been conducted, these measures could be a valid means for interpreting changes in performance and subsequently individualising recovery strategies according to a more accurate account of the match loads imposed on players [28]. Indeed, recent research using global positioning systems $(10 \mathrm{~Hz}$ sampling $)$ in elite players has shown that the distance covered in rapid acceleration/deceleration phases is potentially more sensitive than high-speed distance alone in detecting variations in match running performance [29]. However, the results from this study should be interpreted with caution due to ongoing concerns regarding the validity and accuracy of the GPS technology for measuring such activities [30].

Recent research conducted in elite youth and senior soccer match-play has nevertheless investigated these alternative movement variables that are taxing metabolically and contribute greatly to muscle damage $[16,17]$. For instance, the frequency of rapid accelerations performed per minute markedly declined at the end of a congested period in elite youth players (five matches in $72 \mathrm{~h}$ ) [16]. In contrast, neither total distance covered nor that at high speeds varied. In elite players, a holistic approach was undertaken by linking timemotion data with post-match fatigue obtained using ratings of perceived exertion, creatine kinase concentrations and physical test performance sampled over a 72-h period following match-play [17]. Large magnitude correlations $(r>0.50)$ were reported between the number of short sprints performed and perceived post-match muscle soreness (48 and $72 \mathrm{~h}$ ) as well as the number of rapid changes in direction and decrements in jump performance (24 h).

\subsection{A Holistic Approach to Research into the Effects of Match Congestion}

We suggest that the aforementioned measures of running activities should be part of a more holistic approach to quantify and aid interpretation of potential changes in match performance across congested periods. The inclusion of skill-related data (e.g., frequency and completion rates in technical actions) is necessary; however, only two studies to date have reported such information [9, 15]. Similarly, only one report has considered match performance prior to, during and following periods of fixture congestion [10]. This information would provide additional evidence on the effects of fixture congestion in comparison to 'habitual' performance. Interpretations of data should also attempt to account for the number of games already played, travel time to matches and the potentially confounding effects of time delay between successive matches (e.g., 2 vs. 3 days). Inclusion of a detailed temporal breakdown of running activities rather than simply 'global' measures performed over the entire course of matches (e.g., total distance covered over $90 \mathrm{~min}$ ) could provide more accurate insights. For example, is there a tendency for underperformance in running and/or skill-related actions at the start of play and/or in the final $15 \mathrm{~min}$ in the third match played over a 5-day period? Any holistic approach must also take into consideration the aforementioned performance-related and contextual factors recognised as affecting match-play performance.

In addition, while no definitive marker exists for monitoring biochemical, hormonal and immunological responses to exercise [31], there is still potential for research merging this information with external workload information derived from time-motion analyses. First-hand accounts from the players themselves providing subjective ratings of perceived exertion, muscle soreness, wellness and sleep quality could also aid interpretation of variations in match performance.

While the time and cost of developing, implementing, managing and subsequently interpreting data derived from any holistic monitoring approach might be high, we suggest that identification of and subsequent targeted research to track only individuals who are regularly exposed to match congestion through national team obligations and mid-week European competition might be more pertinent and practically easier to implement. This latter suggestion is important and confirms that information is necessary to identify how frequently the same individuals actually participate in and complete matches in their entirety over both short and/or prolonged congested periods.

\section{Post-Match Recovery Strategies and Match Running Performance}

A review has critically examined the post-match recovery strategies employed in contemporary elite soccer [18]. The aim of these strategies is to reduce the magnitude of fatigue and muscle damage following competition, and therefore accelerating recovery time. Yet, discussion on the efficacy of the recovery regimens used between matches on running performance in subsequent competition was omitted [18]. Researchers have nevertheless claimed that these regimens may have contributed to the maintenance of match running 
performance reported during congested periods $[2,9,10$, 14, 15]. However, it would seem that some of these strategies are commonly used by practitioners in an ad hoc nature despite little scientific evidence being available on the underlying mechanisms and, particularly for the purpose of this paper, on the actual outcomes in competition to validate and support their implementation.

\subsection{Are There any Proven Benefits of Post-Match Recovery Strategies on Ensuing Match Performance?}

Recovery strategies such as hydration, diet and sleep are widely considered to be effective strategies for aiding recovery in elite soccer players [18] and athletes generally [32]. In contemporary elite soccer, additional recovery strategies such as hydrotherapy, compression garments, massage and electrical stimulation are routinely implemented following competition and training. Yet definitive empirical evidence of the direct effects of isolated or combinations of these post-match recovery strategies on counteracting fatigue and, most importantly, maintaining subsequent competitive performance over periods of match congestion, is currently lacking.

To our knowledge, only one study has attempted to investigate the effect of post-match recovery strategies commonly used in contemporary elite senior soccer on running performance during congested periods [33]. This study evaluated the effects of adherence to post-match recovery treatments including exposure to cold-water immersion $\left(10 \mathrm{~min}\right.$ at $\left.10^{\circ} \mathrm{C}\right)$, lower-limb compression garments (30 min duration) and sports massage (petrissage technique for 45-60 $\mathrm{min}$ ) on running performance during three successive matches played with $48-72 \mathrm{~h}$ recovery. No associations were observed between the overall number of treatments received or the number of cold-water treatments alone and any of the running performance variables across the three matches. The authors concluded that adherence to post-match recovery treatments did not aid players in sustaining running performance during short periods of match congestion. However, no information was provided on the effects (e.g., beneficial/harmful) of the recovery intervention determined by analysis of the smallest 'worthwhile' change in match performance indices [23]. Indeed, there is evidence that some practices (e.g., active recovery, cold-water immersion) can be detrimental to recovery in elite athletic populations $[18,32]$. It is also difficult to draw definite conclusions (due to the varying effectiveness previously reported for the treatments) as some players in the above study may or may not have chosen to participate in more than one treatment type.
Research on elite senior Australian Rules Football [34] demonstrated that post-match recovery strategies were not positively associated with match performance (determined subjectively via ratings given by coaching staff). In contrast, perceptual recovery among players was enhanced post-match following the treatments. Finally, research conducted in elite youth soccer players has reported that post-match recovery interventions (e.g., sauna, cold-water immersion, jacuzzi) were beneficial in attenuating decrements in running performance, notably high-speed activity across consecutive matches played within a tight timeframe $[35,36]$. However, the large inter-individual differences in the responses to the treatment suggest that not all players benefited from post-match recovery interventions to the same extent [35].

\subsection{Concerns with Conducting Research on the Benefits of Post-Match Recovery Strategies}

The aforementioned lack of studies addressing this issue is in our opinion mainly related to the problems commonly encountered in conducting controlled intervention studies in elite soccer settings and the large variability inherent in match performance metrics. Difficulties are due to the scheduling of matches/training sessions, gaining access to first-team players and because the use of control versus intervention groups in which only a single population benefits from an intervention is generally unfeasible at the elite level. Furthermore, the transfer of quantifiable outcomes after any form of 'sports science' intervention into meaningful changes in match running performance remains a challenge in elite soccer. The interpretation process of any worthwhile change in performance is generally arbitrary as this is linked to the noisy data due to the unpredictable nature of play and the numerous external factors that dictate running performance $[20,21]$.

Issues surrounding study design also warrant discussion, especially in the absence of a clear set of guidelines for evidence-based recovery practices in elite soccer. In addition, the timing of participation in post-match recovery procedures must be considered (e.g., immediately after play and/or morning after). In water immersion therapy alone, practitioners must consider water temperature (e.g., same/contrasting), delay between therapies, and full or partial immersion of the body [37]. Finally, there are potentially confounding lifestyle factors that are difficult to control, such as compliance with recommended rehydration and nutrition guidelines to ensure restoration of fluids/ energy stores as well as the quantity and quality of sleep between matches. 


\subsection{Future Directions for Research}

Despite the current lack of evidence regarding the effectiveness of post-match recovery interventions, research will no doubt continue to explore the relationship between recovery and competitive performance. Controlled experimental simulations of match-play activity [38] to reproduce the intermittent-endurance demands of competition have received little attention and might be an alternative research avenue to verify post-match fatigue kinetics and their response to different recovery strategies. Randomised controlled, crossover experimental designs using match simulations that resemble typical competitive schedules are necessary to examine the influence of different recovery interventions (single and combinations of treatments) on the accumulation of fatigue and muscle damage. Current studies only tend to analyse the 'effects' after a single competitive match and multiple instances of performance are necessary [8]. Verification of whether the magnitude of neuromuscular and biochemical responses from match to match varies across short- versus long-term periods of fixture congestion is necessary. Varying the content and intensity of successive simulations using time-motion data derived from actual analyses of match-play would also aid replication of real-world competition circumstances.

Match simulations should also incorporate directional changes, accelerations and decelerations, and jumps to increase ecological validity. Indeed, a 90-min intermittent treadmill protocol simulating match-play did not affect peak isometric force production, vertical jump, or single or repeated sprint performance immediately after or throughout the ensuing $72 \mathrm{~h}$ [39]. This finding was attributed in part to the non-inclusion of these locomotor actions. In addition, simulations including these actions would relate better to the physical tests commonly used to determine the physical impact of competition on the postmatch recovery time course. Measures related to muscle damage and force-generating capacity such as maximal voluntary contraction, jump height and short sprint speed are often employed.

\section{Conclusion}

As part of the contemporary preparation process for elite soccer, running performance is frequently monitored using time-motion analyses of match-play in an attempt to determine how players cope physically during periods of fixture congestion. In this article, we recommended a need for factual information on player participation and rotation to identify the extent to which players are actually exposed to fixture congestion. Quantification of the number of occasions in which players compete in successive matches with a minimum $48 \mathrm{~h}$ or less recovery would be a useful starting point, especially as regards the necessity for postmatch recovery interventions. Changes in the design of time-motion studies, notably using a more holistic approach to monitoring and larger sample sizes, are also recommended. While challenging, such an approach would certainly aid interpretation of the potential deleterious effects of fixture congestion on match-to-match performance. Finally, practitioners commonly employ various recovery modalities in an attempt to enhance recovery between successive matches. Yet, scientific evidence available to support their effectiveness in maintaining running performance during fixture congestion is scarce and reflects to some extent the limitations inherent in conducting studies of this nature. We nevertheless recommend that additional experimental investigations, notably using controlled match-play simulations, are attempted.

Acknowledgments The authors have no potential conflicts of interest and no funding was obtained for the preparation of this article.

\section{References}

1. Strudwick T. Contemporary issues in the physical preparation of elite players. In: Williams A, editor. Science and soccer: developing elite performers. London: Routledge; 2012. p. 335-56.

2. Dupont G, Nedelec M, McCall A, et al. Effect of 2 soccer matches in a week on physical performance and injury rate. Am J Sports Med. 2010;38:1752-8.

3. Nédélec M, McCall A, Carling C, et al. Recovery in soccer: part I -post-match fatigue and time course of recovery. Sports Med. 2013;42:997-1015.

4. Fatouros IG, Chatzinikolaou A, Douroudos II, et al. Time-course of changes in oxidative stress and antioxidant status responses following a soccer game. J Strength Cond Res. 2010;24:3278-86.

5. Ispirlidis I, Fatouros IG, Jamurtas AZ, et al. Time-course of changes in inflammatory and performance responses following a soccer game. Clin J Sport Med. 2008;18:423-31.

6. Ascensão A1, Rebelo A, Oliveira E, et al. Biochemical impact of a soccer match - analysis of oxidative stress and muscle damage markers throughout recovery. Clin Biochem. 2008;41:841-51.

7. Ascensão A, Leite M, Rebelo AN, et al. Effects of cold water immersion on the recovery of physical performance and muscle damage following a one-off soccer match. J Sports Sci. 2011;29:217-25.

8. Rollo I, Impellizzeri FM, Zago M, et al. Effects of 1 versus 2 games a week on physical and subjective scores of subelite soccer players. Int J Sports Physiol Perform. 2014;9:425-31.

9. Carling C, Dupont G. Are declines in physical performance associated with a reduction in skill-related performance during elite soccer match-play? J Sports Sci. 2011;29:63-71.

10. Carling C, Le Gall F, Dupont G. Are physical performance and injury risk in a professional soccer team in match play affected over a prolonged period of fixture congestion? Int J Sports Med. 2011;32:1-7.

11. Odetoyinbo K, Wooster B, Lane A. The effect of a succession of matches on the activity profiles of professional soccer players. In: Reilly T, Korkusuz F, editors. Science and football VI. London: Routledge; 2007. p. 105-8. 
12. Rey E, Lago-Peñas C, Lago-Ballesteros J, et al. The effects of a congested fixture period on the activity of elite soccer players. Biol Sport. 2010;27:181-5.

13. Lago-Peñas C, Rey E, Lago-Ballesteros J, et al. The influence of a congested calendar on physical performance in elite soccer. J Strength Cond Res. 2011;25:2111-7.

14. Djaoui L, Wong DP, Pialoux V, et al. Physical activity during a prolonged congested period in a top-class European football team. Asian J Sports Med. 2014;5:47-53.

15. Dellal A, Lago-Peñas C, Rey E, et al. The effects of a congested fixture period on physical performance, technical activity and injury rate during matches in a professional soccer team. Br J Sports Med. Epub 2013 Feb 25.

16. Arruda AFS, Carling C, Zanetti V, et al. Effects of a very congested match schedule on body load impacts, accelerations, and running measures in youth soccer players. Int J Sports Physiol Perform. Epub 2014 Aug 12.

17. Nédélec M, McCall A, Carling C, et al. The influence of soccer playing actions on the recovery kinetics after a soccer match. J Strength Cond Res. 2014;28:1517-23.

18. Nédélec M, McCall A, Carling C, et al. Recovery in soccer: part II-recovery strategies. Sports Med. 2013;43:9-22.

19. Rampinini E, Bosio A, Ferraresi I, et al. Match-related fatigue in soccer players. Med Sci Sports Exerc. 2011;43:2161-70.

20. Carling C. Interpreting physical performance in professional soccer match-play: should we be more pragmatic in our approach? Sports Med. 2013;43:655-63.

21. Gregson W, Drust B, Atkinson G, et al. Match-to-match variability of high-speed activities in premier league soccer. Int $\mathbf{J}$ Sports Med. 2010;31:237-42.

22. Bradley PS, Noakes TD. Match running performance fluctuations in elite soccer: indicative of fatigue, pacing or situational influences? J Sports Sci. 2013;31:1627-38.

23. Hopkins WG, Marshall SW, Batterham AM, et al. Progressive statistics for studies in sports medicine and exercise science. Med Sci Sports Exerc. 2009;41:3-13.

24. Carling C, Wright C, Nelson L, et al. Comment on 'performance analysis in football: a critical review and implications for future research'. J Sports Sci. 2014;32:2-7.

25. Lovell R, Abt G. Individualization of time-motion analysis: a case-cohort example. Int J Sports Physiol Perform. 2013;8:456-8.

26. Hunter F, Bray J, Towlson C, et al. Individualisation of timemotion analysis: a method comparison and case report series. Int J Sports Med. 2015;36(1):41-8.
27. Osgnach C, Poser S, Bernardini R, et al. Energy cost and metabolic power in elite soccer: a new match analysis approach. Med Sci Sports Exerc. 2010;42:170-8.

28. Gaudino P, Iaia FM, Alberti G, et al. Monitoring training in elite soccer players: systematic bias between running speed and metabolic power data. Int J Sports Med. 2013;34:963-8.

29. Akenhead R, Hayes PR, Thompson KG, et al. Diminutions of acceleration and deceleration output during professional football match play. J Sci Med Sport. 2013;16:556-61.

30. Varley MC, Fairweather IH, Aughey RJ. Validity and reliability of GPS for measuring instantaneous velocity during acceleration, deceleration, and constant motion. J Sports Sci. 2012;30:121-7.

31. Halson SL. Monitoring training load to understand fatigue in athletes. Sports Med. 2014;44(Suppl 2):S139-47.

32. Robson-Ansley PJ, Gleeson M, Ansley L. Fatigue management in the preparation of Olympic athletes. J Sports Sci. 2009;27: 1409-20.

33. Carling C, Le Gall F, Cuvelier M, et al. Does adherence to postmatch recovery treatments aid professional soccer players in maintaining physical performance during periods of fixture congestion? 3rd World conference on science and soccer; 14-16 May 2012. Ghent: University Press; Abstract book 101. p. 161.

34. Bahnert A, Norton K, Lock P. Association between post-game recovery protocols, physical and perceived recovery, and performance in elite Australian Football League players. J Sci Med Sport. 2013;16:151-6.

35. Buchheit M, Horobeanu C, Mendez-Villanueva A, et al. Effects of age and spa treatment on match running performance over two consecutive games in highly trained young soccer players. J Sports Sci. 2011;29:591-8.

36. Rowsell GJ, Coutts AJ, Reaburn P, et al. Effect of post-game cold-water immersion on subsequent match running performance in junior soccer players during tournament play. J Sports Sci. 2011;29:1-6.

37. Versey NG, Halson SL, Dawson BT. Water immersion recovery for athletes: effect on exercise performance and practical recommendations. Sports Med. 2013;43:1101-30.

38. Ingram J, Dawson B, Goodman C, et al. Effect of water immersion methods on post-exercise recovery from simulated team sport exercise. J Sci Med Sport. 2009;12:417-21.

39. Nedelec M, Wisloff U, McCall A, et al. Recovery after an intermittent test. Int J Sports Med. 2013;34:554-8. 